$(\mathrm{p}<0.001)$ whereas G1407 genogroup was associated with $\mathrm{MIC}=0.125 \mathrm{mg} / \mathrm{L}$ for CFX $(\mathrm{p}=0.001)$, resistance to CIP, PEN $(p=0.016)$ and TET $(p<0.001)$. Isolates that shared the same tbpB 29 allele were associated with resistance to AZT $(p=0.008)$ and PEN $(p=0.035)$. ST338 was associated with bla $a_{\text {TEM-1 }}$ gene $(\mathrm{p}<0.001)$ and tet $\mathrm{M}(\mathrm{p}=0.006)$, whereas the cluster sharing the same $t b p \mathrm{~B} 137$ allele was associated with tet $\mathrm{M}$ gene.

Conclusion This study showed high rates of resistance to PEN, TET and CIP associated with persistence and dissemination of gonococcal strains resistant to CIP and plasmid resistance to PEN and TET. Despite the high resistance profile to CIP, the treatment recommended to the south region of Brazil was the association of CIP-AZT until 2017, when the national recommendation changed it to CRO-AZT.

Disclosure No significant relationships.

\section{P656 GYRA AND PARC MUTATIONS IN FLUOROQUINOLONE- RESISTANT NEISSERIA GONORRHOEAE ISOLATES FROM KENYA}

\footnotetext{
${ }^{1}$ Mary Kivata*, ${ }^{2}$ Margaret Mbuchi, ${ }^{2}$ Fredrick Eyase, ${ }^{2}$ Wallace Bulimo, ${ }^{2}$ Cecilia Kyanya, ${ }^{2}$ Valerie Oundo, ${ }^{2}$ Simon Muriithi, ${ }^{2}$ Ben Andagalu, ${ }^{3}$ Wilton Mbinda, ${ }^{4}$ Olusegun Soge, ${ }^{5} \mathrm{R}$ Mcclelland, ${ }^{2}$ Willy Sang Sang, ${ }^{2}$ James Mancuso. ${ }^{1} J$ omo Kenyatta University of Agriculture and Technology, Institute for Biotechnology Research, Thika, Kenya; ${ }^{2}$ US Army Medical Research Directorate-Kenya, Village Market, Nairobi, Kenya; ${ }^{3}$ Karatina University, Karatina, Kenya; ${ }^{4}$ University of Washington, Global Health, Seattle, USA; ${ }^{5}$ University of Washington, Epidemiology, Seattle, USA
}

\subsection{6/sextrans-2019-sti.724}

Background Phenotypic fluoroquinolone resistance was first reported in Western Kenya in 2009 and later in Coastal Kenya and Nairobi. Until recently gonococcal fluoroquinolone resistance mechanisms in Kenya had not been elucidated. The aim of this paper is to analyze mutations in both GyrA and ParC responsible for elevated fluoroquinolone MICs in Neisseria gonorrhoeae (GC) isolated from heterosexual individuals from different locations in Kenya.

Methods Antimicrobial Susceptibility Tests were done on 84 GC in an ongoing STI surveillance program. Of the 84 isolates, 22 resistant to two or more classes of antimicrobials were chosen for analysis. Antimicrobial susceptibility tests were done using E-test and the results were interpreted with reference to European Committee on Antimicrobial Susceptibility Testing (EUCAST) standards. The isolates were sub-cultured and whole genomes sequenced using Illumina platform. Reads were assembled de novo using Velvet, and mutations in the GC Quinolone Resistant Determining Regions identified using Bioedit sequence alignment editor. Single Nucleotide Polymorphism based phylogeny was inferred using RaxML.

Results Double GyrA mutations; S91F and D95G/D95A were identified in 20 isolates. Of these 20 isolates, 14 had an additional E91G ParC mutation and significantly higher ciprofloxacin MICs $(p=0.0044 \%)$. On the contrary, norfloxacin MICs of isolates expressing both GyrA and ParC QRDR mutations were not significantly high $(p=0.82)$ compared to MICs of isolates expressing GyrA mutations alone. No single GyrA mutation was found in the analyzed isolates, and no isolate contained a ParC mutation without the simultaneous presence of double GyrA mutations. Maximum likelihood tree clustered the 22 isolates into 6 distinct clades.

Conclusion Simultaneous presence of mutations in ParC and GyrA has been reported to increase gonococcal fluoroquinolone resistance from different regions in the world. Our findings indicate that GyrAS91F, D95G/D95A and ParC E91G amino acid substitutions mediate high fluoroquinolone resistance in the analyzed Kenyan GC.

Disclosure No significant relationships.

\section{P657 HIGH AZITHROMYCIN RESISTANCE AND DECREASING CEFTRIAXONE SUSCEPTIBILITY IN NEISSERIA GONORRHOEAE IN SHENZHEN (2010-2017), CHINA}

${ }^{1}$ Feng Wang ${ }^{*},{ }^{1}$ Yizhun Li, ${ }^{2}$ Lijun Zhang, ${ }^{3}$ Jing Huang, ${ }^{4}$ Yueping Yin. ${ }^{1}$ Shenzhen Center for Chronic Disease Control, STD Control Laboratory, Shenzhen, China; '2Shenzhen Maternal and Children Health Care Hospital, Shenzhen, China; ${ }^{3}$ Shenyang Pharmaceutical University, Shenyang, China; ${ }^{4}$ National Center for STD Control, Chinese Center for Disease Control and Prevention; Institute of Dermatology, Chinese Academy of Medical Sciences and Peking Union Medical College, Nanjing, China

\subsection{6/sextrans-2019-sti.725}

Background Emergence and development of resistance in Neisseria gonorrhoeae (NG) to antibiotics has become a major public health problem worldwide. The aim of our study was to determine the antimicrobial susceptibility, especially ceftriaxone and azithromycin, and molecular epidemiological typing of NG isolates in Shenzhen, China.

Methods A total of 1,282 NG isolates were collected from Shenzhen, between 2010 and 2017. The minimum inhibitory concentrations (MICs) of NG isolates were determined by the agar dilution method. Resistance to azithromycin (AZM-R) was defined as MIC $\geq 1.0 \mathrm{mg} / \mathrm{L}$, and decreased susceptibility to ceftriaxone $\left(\mathrm{CRO}^{\mathrm{D}}\right)$ was defined as $\mathrm{MIC} \geq 0.125 \mathrm{mg} / \mathrm{L}$. Isolates were genotyped using Neisseria gonorrhoeae multi-antigen sequence typing (NG-MAST).

Results Among the isolates, 5.0\% showed $\mathrm{CRO}^{\mathrm{D}}$, $17.3 \%$ was AZM-R and $1.3 \%$ was both $\mathrm{CRO}^{\mathrm{D}}$ and AZM-R. Increasing ceftriaxone MICs was found from 2010-2014 to 2015-2017 [ridit value: $0.585 ; 95 \%$ confidence interval $(95 \% \mathrm{CI})$ : 0.559 0.611], but not for $\mathrm{CRO}^{\mathrm{D}}$ and AZM-R. The proportions of

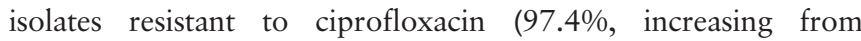
$96.6 \%$ in $2010-2014$ to $98.8 \%$ in $2015-2017$ ) and penicillin (68.2\%, increasing from $60.6 \%$ in $2013-2014$ to $73.5 \%$ in 2015-2017) were increasing, but PPNG was decreased. All isolates were susceptible to spectinomycin. Genogroup 5308 was inversely association associated with AZM-R; the corresponding odds ratio (95\%CI) was: 0.098 (0.013-0.747).

Conclusion The founding of increasing ceftriaxone MICs over the time and high proportions of AZM-R in Shenzhen region calls for reevaluation dual therapy. Timely and efficient surveillance with isolates characterization and changes of susceptibility over time is essential.

Disclosure No significant relationships. 